\title{
Erratum to: A Framework for Soft Sensing of Liquid Pool Length of Continuous Casting Round Blooms
}

JIAN YANG, ZHI XIE, JING NING, WENHONG LIU, and ZHENPING JI

DOI: $10.1007 / \mathrm{s} 11663-014-0225-6$

(C) The Minerals, Metals \& Materials Society and ASM International 2014

\section{Erratum to: METALLURGICAL AND MATERIALS} TRANSACTIONS B, 2014, Volume 45, Number 4, pp. 1545-1556 DOI: 10.1007/s11663-014-0079-y

EQ. (40) in page 1554 should be:

$$
H_{\alpha, c}=\left\{\begin{array}{l}
1000 \cdot\left(5188 T(K)^{-1}-86+0.505 T(K)\right. \\
\left.-6.55 \cdot 10^{-5} T(K)^{2}+1.5 \cdot 10^{-7} T(K)^{3}\right) \quad T(K) \leq 800 \\
1000 \cdot\left(-1.11 \cdot 10^{6} T(K)^{-1}+4056-4.72 T(K)\right. \\
\left.+2.292 \cdot 10^{-3} T(K)^{2}\right) \quad 800<T(K) \leq 1000 \\
1000 \cdot\left(5780-11.5 T(K)+6.238 \cdot 10^{-3} T(K)^{2}\right) \\
1000<T(K) \leq 1042 \\
1000 \cdot\left(-18379+34.87 T(K)-0.016013 T(K)^{2}\right) \\
1042 \leq T(K) \leq 1060 \\
1000 \cdot\left(-5.21766 \cdot 10^{6} T(K)^{-1}+12822-10.068 T(K)\right. \\
\left.+2.9934 \cdot 10^{-3} T(K)^{2}\right) \quad 1060<T(K) \leq 1184
\end{array}\right.
$$

$$
H_{\alpha, c}=\left\{\begin{array}{l}
1000 \cdot\left(5188 T(K)^{-1}-86+0.505 T(K)\right. \\
\left.-6.55 \cdot 10^{-5} T(K)^{2}+1.5 \cdot 10^{-7} T(K)^{3}\right) \quad T(K) \leq 800 \\
1000 \cdot\left(-1.11 \cdot 10^{6} T(K)^{-1}+4056-4.72 T(K)\right. \\
\left.+2.292 \cdot 10^{-3} T(K)^{2}\right) \quad 800<T(K) \leq 1000 \\
1000 \cdot\left(\mathbf{5 7 2 0}-11.5 T(K)+6.238 \cdot 10^{-3} T(K)^{2}\right) \\
\quad 1000<T(K) \leq 1042 \\
1000 \cdot\left(-\mathbf{1 8 . 3 7 9}+34.87 T(K)-0.016013 T(K)^{2}\right) \\
1042 \leq T(K) \leq 1060 \\
1000 \cdot\left(-5.21766 \cdot 10^{6} T(K)^{-1}+\mathbf{1 2 . 8 2 2}-10.068 T(K)\right. \\
\left.+2.9934 \cdot 10^{-3} T(K)^{2}\right) \quad 1060<T(K) \leq 1184
\end{array}\right.
$$

instead of:

JIAN YANG, Ph.D. Student, ZHI XIE and JING NING, Professors, are with the College of Information Science and Engineering, Northeastern University at Shenyang, Liaoning, P.R. China 110819. Contact e-mail: neuyangjian@163.com WENHONG LIU, Associate Professor, is with State Key Laboratory of Synthetical Automation for Process Industries, Northeastern University at Shenyang, Liaoning, P.R. China 110819. ZHENPING JI, Professor, is with the College of Information Science and Engineering, Shenyang Ligong University at Shenyang, P.R. China 110159.

The online version of the original article can be found under doi: 10.1007/s11663-014-0079-y.

Article published online October 16, 2014. 